\title{
PAPERS
}

\section{Effect of atropine on gastro-oesophageal reflux and transient lower oesophageal sphincter relaxations in patients with gastro-oesophageal reflux disease}

\author{
I Lidums, H Checklin, R K Mittal, R H Holloway
}

\begin{abstract}
Background-Atropine reduces the rate of reflux episodes in normal subjects by inhibition of transient lower oesophageal sphincter (LOS) relaxations. The aim of this study was to investigate the effect of atropine on the rate and mechanisms of reflux in patients with reflux disease.

Methods-Oesophageal motility and pH were recorded for one hour after a meal in 15 patients with reflux disease. On separate days, atropine $(15 \mu \mathrm{g} / \mathrm{kg}$ bolus intravenously, $4 \mu \mathrm{g} / \mathrm{kg} / \mathrm{h}$ infusion) or saline were given and maintained for the recording period.

Results-Atropine significantly reduced basal LOS pressure from $7.1(2.2)$ to 2.9 (1.3) $\mathrm{mm} \mathrm{Hg}$ (mean (SEM)). Atropine also reduced the rate of reflux episodes from $5.0(2.0-8.75)$ to $1.0(0-6.25)$ per hour (median (interquartile range)) largely because of a decrease in the rate of transient LOS relaxations from $2.0(0-4.75)$ to 0 (0-0) per hour and abolition of reflux during swallow induced LOS relaxation. There was no change in the rate of reflux episodes because of absent basal LOS pressure.

Conclusions-Atropine inhibits reflux in patients with reflux disease largely by inhibition of transient LOS relaxations and swallow induced LOS relaxation. These findings suggest that pharmacological control of reflux through control of transient LOS relaxations is possible in patients with reflux disease.

(Gut 1998;43:12-16)
\end{abstract}

Division, University of Virginia Health

Sciences Center, Charlottesville, VA, USA

R K Mittal

Correspondence to: Dr R Holloway, Department of Gastrointestinal Medicine, Royal Adelaide Hospital, North Terrace, Adelaide, SA 5000, Australia.

Accepted for publication 4 February 1998 pression of the LOS by the crural diaphragm constitute the principal mechanism preventing the reflux of gastric contents into the oesophathe key determinant of gastro-oesophageal gus. Incompetence of this anti-reflux barrier is reflux. In normal subjects, reflux occurs almost exclusively during transient LOS relaxation. ${ }^{12}$ In patients with gastro-oesophageal reflux disease, most reflux episodes also occur during transient LOS relaxations, ${ }^{3-5}$ although low basal LOS pressure is the predominant mechanism in some patients. ${ }^{6}$

We have shown recently that atropine substantially inhibits gastro-oesophageal reflux in normal subjects by inhibition of transient LOS relaxations. ${ }^{7}$ Previous studies on the effects of atropine on reflux in patients with reflux disease are few and the results conflicting. Atropine has been reported to increase the incidence of reflux during abdominal compression, ${ }^{8}$ but decrease the rate of reflux episodes during postprandial oesophageal $\mathrm{pH}$ monitoring. ${ }^{9}$ Neither of these studies, however, examined reflux mechanisms. The aim of this study therefore was to investigate the effects of atropine on the rate and mechanisms of reflux in patients with gastro-oesophageal reflux disease.

\section{Methods}

SUBJECTS

Studies were performed in 15 patients (11 men, four women) with gastro-oesophageal reflux disease defined as either endoscopy proven erosive or ulcerative oesophagitis (grade 2 in 10 patients, grade 4 in one patient), ${ }^{10}$ or excessive oesophageal acid exposure $(\mathrm{pH}<4$ for more than $5 \%$ of total time) on 24 hour ambulatory $\mathrm{pH}$ monitoring (four patients). The median age of the patients was 48 years (range 32 to 69 years). Eight patients had a hiatus hernia proven endoscopically or during oesophageal manometry; in two patients the hernia was more than $5 \mathrm{~cm}$ in length. Patients with a history of previous gastric surgery or other systemic disease known to influence reflux such as scleroderma were excluded from the study. None of the patients was taking medications known to influence oesophageal motor function, and acid suppression medications were stopped at least 72 hours before the study. Each subject gave written informed consent, and the protocol was approved by the 


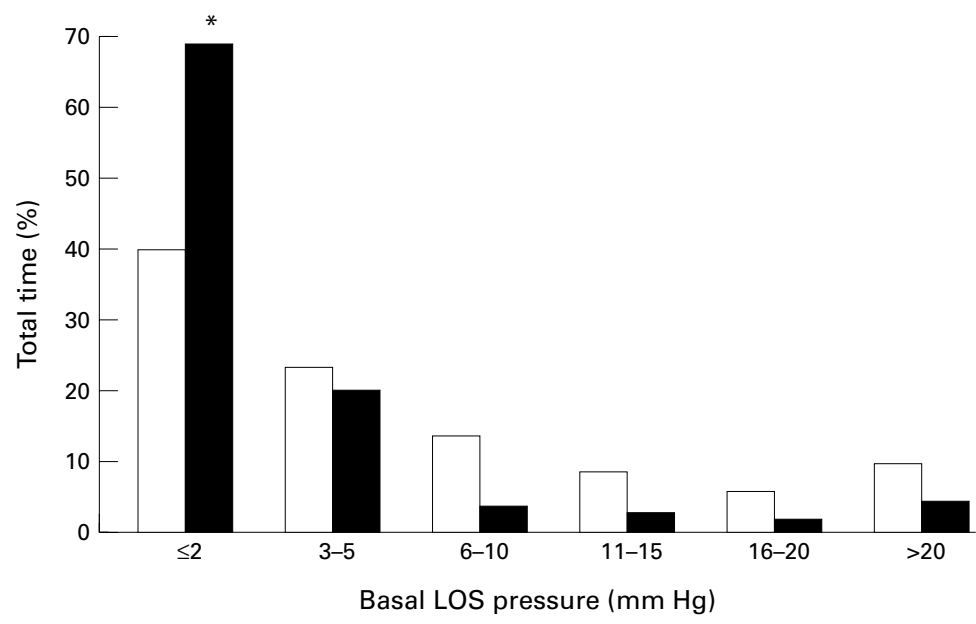

Figure 1 Effect of atropine on lower basal oesophageal sphincter (LOS) pressure. Data have been grouped into intervals of pressure and are expressed as a percentage of the total recording time. Atropine significantly increased the proportion of time that LOS pressure was $2 \mathrm{~mm} \mathrm{Hg}$ or less. ${ }^{\star} p<0.05 v$ control (saline infusion). Open columns, saline; closed columns, atropine.
STUDY PROTOCOL

Subjects were studied after an overnight fast. The manometric assembly and $\mathrm{pH}$ electrodes were passed via the nose. A cannula was inserted into the forearm vein for subsequent administration of saline or atropine. The subjects were allowed to accommodate to the assembly for 10 minutes while in the right lateral position. They then sat up and ate a 3000 $\mathrm{kJ}$ (750 kcal) soft mixed nutrient meal, consisting of savoury minced meat, mashed vegetables, $150 \mathrm{ml}$ milk, and ice cream. Thirty minutes after the meal the subjects lay on a bed in the right lateral position. On separate days, intravenous atropine $(15 \mu \mathrm{g} / \mathrm{kg}$ bolus, $4 \mu \mathrm{g} / \mathrm{kg} / \mathrm{h}$ infusion) or saline were then given in randomised order and maintained for the subsequent 60 minute recording period. During the infusion period, the pulse and blood pressure were recorded every 15 minutes, and the subjects were asked about symptoms such as blurred vision and dry mouth. Because of the potential for atropine to impair oesophageal acid clearance, thereby making it difficult to identify reflux episodes, a $5 \mathrm{ml}$ oral bolus of $1.4 \%$ bicarbonate was given if oesophageal $\mathrm{pH}$ did not return to a value above 4 within two minutes of an acid reflux event in order to aid oesophageal clearance of the acid refluxate. Ten water swallows of a $5 \mathrm{ml}$ bolus were performed at the end of the 60 minute recording period. The atropine and saline placebo were given on separate days in a randomised order at least one week apart.

\section{DATA ANALYSIS}

Basal LOS pressure was measured at end expiration and referenced to intragastric pressure. A visual mean was taken at one minute intervals and a grand mean for the 60 minutes recording period was calculated.

Acid reflux was defined as a fall in oesophageal $\mathrm{pH}$ below 4 for at least four seconds or, if basal oesophageal $\mathrm{pH}$ was already below 4 , a further fall in $\mathrm{pH}$ of at least $1 \mathrm{pH}$ unit. The onset of the fall in oesophageal $\mathrm{pH}$ was used as the reference time for analysis of the motor events associated with reflux. For each reflux episode, the mechanism of reflux was determined from the pattern of LOS pressure and oesophageal body activity and their relationship to swallowing, and the occurrence of abdominal straining. ${ }^{56}$ Transient LOS relaxations were defined and counted separately according to criteria published previously. ${ }^{12}$ In addition, when basal LOS pressure was $2 \mathrm{~mm}$ $\mathrm{Hg}$ or less, the LOS pressure tracing was analysed for episodes of transient inhibition of the crural diaphragm as evidenced by loss of respiratory increases in LOS pressure recorded by the reverse perfused sleeve sensor. These episodes were taken as surrogate markers of transient LOS relaxations. ${ }^{73}$

The rate of spontaneous swallowing was determined by counting the pharyngeal pressure waves. Primary peristalsis in response to the ten water swallows was analysed for success rate, mean amplitude, and peristaltic velocity. Primary peristalsis was defined as successful if the pressure wave amplitude was more than 12
Figure 2 Effect of atropine on the number of reflux episodes. Data are depicted as median (interquartile range). ${ }^{\star} p<0.05 v$ control (saline infusion). 


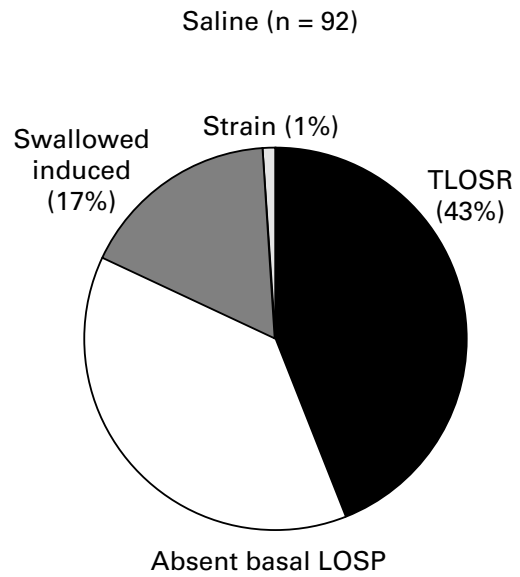

$(38 \%)$

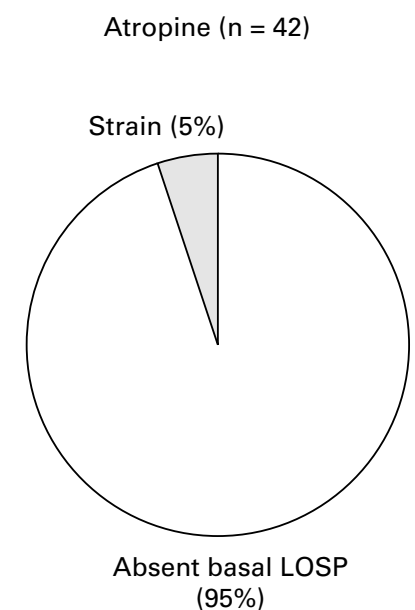

Figure 3 Mechanism of reflux during saline and atropine infusion. The data for each group have been pooled and the numbers in parentheses indicate the percentage of reflux episodes. LOSP, lower oesophageal sphincter pressure; TLOSR, transient LOS relaxation.

$\mathrm{mm} \mathrm{Hg}$ in the proximal oesophagus and more than $25 \mathrm{~mm} \mathrm{Hg}$ in the distal oesophagus, and peristaltic velocity between adjacent oesophageal recording sites was less than $6 \mathrm{~cm} / \mathrm{s}^{2}$

Data for reflux episodes and transient LOS relaxations were analysed using the Wilcoxon signed rank test and presented as median (interquartile range). All other data were analysed using the paired $t$ test and are presented as mean (SEM). p $<0.05$ was accepted as indicating statistical significance.

\section{Results}

All patients reported symptoms and exhibited signs of cholinergic blockade during atropine infusion. The mean pulse rate during atropine (98 (2) beats/min) was significantly higher than that during saline infusion (74 (2) beats/min; $\mathrm{p}<0.001)$. The rate of swallowing during atropine infusion $(84(2) / \mathrm{h})$ was similar to that during saline infusion $(77(9) / \mathrm{h} ; \mathrm{p}=0.5)$.

BASAL LOS PRESSURE

Atropine significantly reduced mean basal LOS pressure from 7.1 (2.2) to 2.9 (1.3) $\mathrm{mm}$ $\mathrm{Hg}(\mathrm{p}<0.01)$ and increased the proportion of

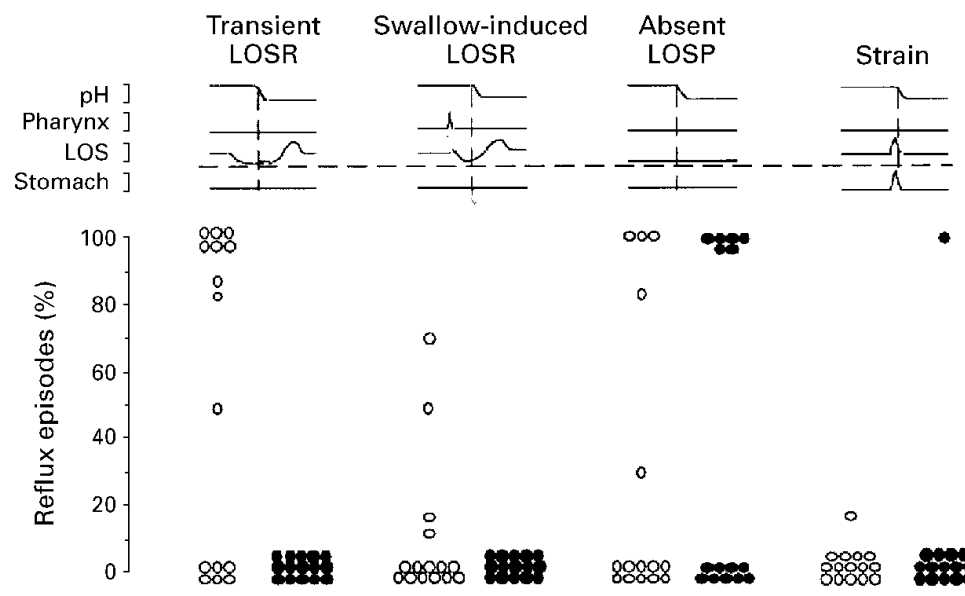

Figure 4 Effect of atropine on the mechanism of reflux. Each point represents the proportion of reflux episodes in an individual patient that is attributable to a particular mechanism. LOSR, lower oesophageal sphincter relaxation; LOSP, lower oesophageal sphincter pressure. Open circles, saline; closed circles, atropine.

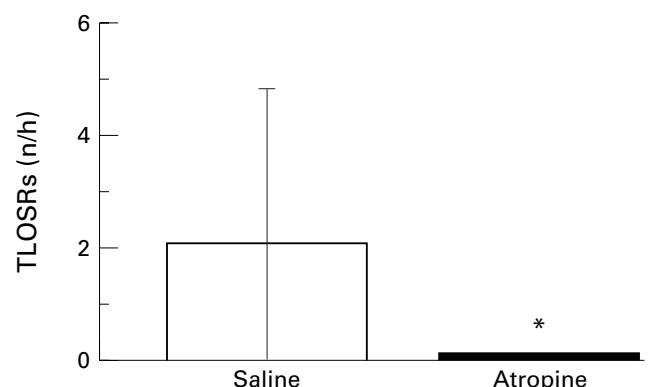

Figure 5 Effect of atropine on the number of transient lower oesophageal sphincter relaxations (TLOSRs). The data are depicted as median and interquartile range. ${ }^{*} p<0.01 v$ control (saline infusion).

time that LOS pressure was $2 \mathrm{~mm} \mathrm{Hg}$ or less from $40(9)$ to $69(9) \%(\mathrm{p}<0.05)$ (fig 1$)$.

GASTRO-OESOPHAGEAL REFLUX

Atropine significantly reduced the frequency of reflux episodes from a median of 5.0 (2.08.75) per hour to $1.0(0-6.25)$ per hour $(\mathrm{p}<0.05)$ (fig 2). During the saline infusion, 92 reflux episodes were scored in the 15 subjects. Overall, the predominant reflux mechanisms were transient LOS relaxations (40 episodes) and absent basal LOS pressure (35 episodes); 16 reflux episodes occurred during swallow induced LOS relaxation and one during straining (fig 3). However, there was heterogeneity among the patients with regard to the predominant mechanism of reflux; eight patients refluxed almost exclusively during transient LOS relaxations and four patients almost exclusively during periods of absent basal LOS pressure (fig 4).

During atropine infusion, 42 reflux episodes were scored in the 15 subjects; 40 were due to absent LOS pressure and two were associated with abdominal straining. None of the reflux episodes during atropine infusion could be attributed to transient LOS relaxations or swallow induced LOS relaxation (figs 3 and 4). Of the eight patients who, during saline, refluxed mostly during transient LOS relaxations, four exhibited no reflux and four continued to reflux because of absent basal LOS pressure, as did the patient who refluxed predominantly during swallow induced LOS relaxation during saline infusion. However, atropine inhibited reflux in two of the four patients who refluxed predominantly during periods of absent basal LOS pressure.

TRANSIENT LOS RELAXATION

Atropine significantly reduced the frequency of transient LOS relaxations from a median of 2.0 $(0-4.75)$ per hour to $0(0-0)$ per hour $(\mathrm{p}<0.01)$ (fig 5). This effect remained significant even if the time during which basal LOS pressure was $2 \mathrm{~mm} \mathrm{Hg}$ or less was excluded from the analy-

Table 1 Effect of atropine om primary peristalsis

\begin{tabular}{lll}
\hline & Saline & Atropine \\
\hline Peristaltic success (\%) & $48(9)$ & $9(6)^{\star}$ \\
Distal amplitude (mm Hg) & $55(8)$ & $27(5)^{\star}$ \\
Distal velocity (cm/s) & $2.6(0.2)$ & $3.6(0.4)^{\star}$ \\
\hline
\end{tabular}

Data are expressed as mean (SEM).

${ }^{\star} \mathrm{p}<0.05 v$ saline. 
sis. During saline infusion, there were 49 transient LOS relaxations, of which $40(82 \%)$ were associated with reflux. During atropine infusion, there were only two transient LOS relaxations, both of which occurred in the one patient, neither of which was associated with a reflux event. No episodes of transient inhibition of the crural diaphragm were seen during periods of low $(\leqslant 2 \mathrm{~mm} \mathrm{Hg})$ basal LOS pressure.

PRIMARY PERISTALSIS

Atropine had no effect on the degree of swallow induced LOS relaxation with water swallows. Nadir LOS pressure during saline infusion (1.2 (0.3) $\mathrm{mm} \mathrm{Hg}$ ) was similar to that seen during atropine administration $(0.5(0.3) \mathrm{mm} \mathrm{Hg} ; \mathrm{p}=$ $0.31)$. However, atropine significantly affected primary peristalsis; it reduced the rate of peristaltic success and wave amplitude, and increased peristaltic velocity in the distal oesophagus (table 1 ).

\section{Discussion}

We have shown recently that atropine substantially inhibits reflux in normal subjects by inhibition of transient LOS relaxations. ${ }^{7}$ Whether or not atropine would also inhibit reflux in patients with reflux disease, however, was not clear. Whereas normal subjects reflux almost exclusively during transient LOS relaxations, patients with reflux disease exhibit a greater degree of heterogeneity of reflux mechanisms. Although most patients reflux predominantly during transient LOS relaxations, ${ }^{3-5}$ reflux during periods of absent basal LOS pressure is the most common mechanism of reflux in a minority of patients. ${ }^{6}$ A low basal LOS pressure induced by atropine is insufficient to promote free reflux in healthy subjects. ${ }^{7}$ However, atropine could conceivably aggravate reflux in some patients by reduction of an already low basal LOS pressure to a level below the threshold for gastro-oesophageal competence, or merely alter the mechanism of reflux from transient LOS relaxation to absent basal LOS pressure without changing significantly the overall rate of reflux episodes. The present study has shown that, like the effect in normal subjects, atropine also inhibits reflux in patients with reflux disease largely by inhibition of transient LOS relaxations as well as by inhibition of reflux during swallow induced LOS relaxation.

Atropine almost completely inhibited transient LOS relaxations and totally abolished reflux during both transient and swallow induced LOS relaxations. Although atropine also reduced basal LOS pressure, its effect on transient LOS relaxations cannot be explained merely on the basis of a reduction in basal LOS pressure to a level at which transient LOS relaxations could not be scored or recognised, as the effect was significant even when the time during which basal LOS pressure was absent was excluded from the analysis. Moreover, even during periods of low basal LOS pressure, there were no episodes of transient inhibition of the crural diaphragm, which are useful markers for transient LOS relaxations. ${ }^{7}$ Likewise, the unexpected reduction in reflux during swallow induced LOS relaxation was not due to a reduction in the rate of swallowing.

The inhibitory effect of atropine on reflux in patients with reflux disease was less marked than that seen previously in normal subjects. ${ }^{7}$ The most plausible explanation is that transient LOS relaxations were not the only mechanism of reflux in the patients. As patients with reflux oesophagitis reflux through a variety of mechanisms, one would not expect atropine to reduce reflux in all patients. Atropine may only be expected to reduce the frequency of reflux in those patients who reflux predominantly during transient LOS relaxations and may not be of benefit in those patients who have a very low resting LOS pressure and high degree of free reflux. In keeping with this notion, there was no change in the number of reflux episodes during absent basal LOS pressure, although reflux during transient and swallow induced LOS relaxations was totally abolished. The lack of apparent effect on reflux during absent basal LOS pressure was the result of a change in reflux mechanism from that of transient LOS relaxation to that of absent basal LOS pressure in four patients, balanced partly by the abolition of reflux during absent basal LOS pressure in two patients.

The effect of atropine on basal LOS pressure was more marked in patients with reflux disease than in the previous study in normal subjects. The reasons for this are not clear but may relate to defective vagal cholinergic innervation to the oesophagus. Experimental oesophagitis is associated with impaired cholinergic innervation to the LOS in cats. ${ }^{14}$ About $40 \%$ of patients with reflux disease have abnormal parasympathetic cardiovascular reflexes, ${ }^{15} 16$ and the gastric secretory response to insulin is impaired in some patients with reflux disease, ${ }^{17}$ suggesting abnormal vagal function in these patients.

Reflux was not seen during periods of low basal LOS pressure induced by atropine in normal subjects. This contrasts with our findings in patients with reflux disease, in some of whom atropine provoked reflux by this mechanism. This difference may have resulted from the greater effect of atropine on basal LOS pressure in the reflux patients. However, half of our patients had hiatus hernias. Loss of the extrinsic diaphragmatic support could potentially aggravate the effects of low or absent LOS pressure on gastro-oesophageal competence. ${ }^{18}$

Anticholinergic agents have previously been regarded as being contraindicated in patients who are prone to gastro-oesophageal reflux disease, because of their effect on reducing LOS pressure. ${ }^{19} 20$ Reports on the effect of atropine on reflux, however, have been conflicting. Atropine has been reported not to increase reflux in normal volunteers ${ }^{21}$ or in pregnant women. ${ }^{22}$ Information on the effect of anticholinergic agents on reflux in patients with gastro-oesophageal reflux disease is limited. In a scintigraphic study, atropine has been reported to increase reflux provoked by abdominal compression. ${ }^{8}$ In contrast, a single subcuta- 
neous dose of atropine was found to decrease the frequency of reflux as measured by $\mathrm{pH}$ monitoring during the first postprandial hour. ${ }^{9}$ These inconsistencies are probably a result of differences in methodology, dose, and route of atropine administration among the studies.

The mechanism of action of atropine in reducing gastro-oesophageal reflux and transient LOS relaxations remains speculative at this stage. It is possible that atropine reduces gastric tone and thereby the stimulus to the gastric mechanoreceptors. Atropine decreases proximal gastric tone in the fasting state. ${ }^{23}$ However, although atropine has been reported to have no effect on postprandial proximal gastric tone, ${ }^{24}$ the dose of atropine used was substantially lower than that used in the present study. It is also possible that atropine acts centrally on the integrative mechanisms in the brain stem that are believed to mediate transient LOS relaxations. In support of this hypothesis are our recent observations that atropine also inhibits LOS relaxations induced by pharyngeal stimulation, ${ }^{25}$ and that hyoscine butyl bromide, a quaternary anticholinergic agent that does not cross the blood-brain barrier, does not inhibit transient LOS relaxations in normal subjects. ${ }^{26}$ It seems unlikely, however, that atropine affects the motor limb of the putative vagovagal reflex arc that is believed to mediate transient LOS relaxations, ${ }^{27}$ as, like findings of previous studies ${ }^{20}$, atropine had no effect on swallow induced LOS relaxation. The reduction in reflux during swallow induced LOS relaxation, however, may indicate a more subtle interference with the motor pathway controlling LOS relaxation.

Pharmacological reduction in the rate of transient LOS relaxations is a physiologically attractive approach to the treatment of reflux disease. Although the reduction in reflux episodes was less substantial than that seen in normal subjects, consistent with the previous study in normal subjects there was complete abolition of reflux during transient LOS relaxations. This finding suggests the possibility of pharmacological control of reflux by inhibition of transient LOS relaxations. In patients with reflux disease, the effect of such an approach is likely to be influenced by the mix of reflux mechanisms in individual subjects. However, as most patients with reflux disease have no macroscopic oesophagitis and are therefore likely to reflux predominantly during transient LOS relaxations, ${ }^{5}$ effective pharmacological control of transient LOS relaxations is likely to be useful in most patients. Atropine or other non-selective anticholinergic agents are not themselves appropriate potential therapeutic agents in this regard because of their deleterious effects on basal LOS pressure and oesophageal clearance. However, the findings point the way to development of other drugs that have more selective effects on triggering of transient LOS relaxations.
This work was supported by a project grant from the NHMRC of Australia. I L was supported by a Dawes Scholarship from the Royal Adelaide Hospital. The results were presented at the American Gastroenterological Association Meeting, Washington, USA, in May 1997, and have been published in abstract form in Gastroenterology 1997;112:A775.

1 Dent J, Dodds WJ, Friedman RH, et al. Mechanism of gastroesophagal reflux in recumbent asymptomatic subjects. $\mathcal{F}$ Clin Invest 1980;65:256-67.

2 Schoeman MN, Holloway RH. Stimulation and characteristics of secondary oesophageal peristalsis in normal tics of secondary oesophaged
subjects. Gut 1994;35:152-8.

3 Dodds WJ, Dent J, Hogan WJ, et al. Mechanisms of gastroesophageal reflux in patients with reflux esophagitis. $N$ Engl Med 1982;307:1547-52.

4 Mittal RK, McCallum RW. Characteristics and frequency of transient relaxations of the lower esophageal sphincter on patients with reflux esophagitis. Gastroenterology 1988;95: 593-9.

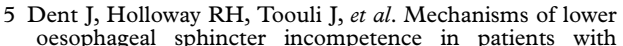
ymptomatic gastroesophageal reflux. Gut 1988;29:10208.

6 Penagini R, Schoeman MN, Dent J, et al. Motor events underlying gastro-oesophageal reflux in ambulant patients with reflux oesophagitis. Neurogastroenterol Motil 1996;8: 131-41.

7 Mittal RK, Holloway R, Dent J. Effect of atropine on the frequency of reflux and transient lower esophageal sphincfrequency of reflux and transient lower esophageal sphinc109:1547-54

8 Fisher RS, Malmud LS, Roberts GS, et al. The lower esophageal sphincter as a barrier to gastroesophageal reflux. Gastroenterology 1977;72:19-22.

9 Pflucke VF, Anders O, Schreiber H. The effect of neostigmine and atropine on gastro-oesophageal reflux. Deutsche Zeitschrift fur Verdaunngs- und Stoffwechselkrankheiten 1981;41:270-5.

10 Hetzel DJ, Dent J, Reed WD, et al. Healing and relapse of severe peptic esophagitis after treatment with omeprazole. Gastroenterology 1988;95:903-12.

11 Sivri B, Mittal RK. Reverse-perfused sleeve: an improved device for measurement of sphincteric function of the crural diaphragm. Gastroenterology 1991;101:962-9.

12 Holloway RH, Penagini R, Ireland AC. Criteria for objective definition of transient lower esophageal sphincter relaxadefinition of transient lower esophage
tion. Am f Physiol 1995;268:G128-33.

tion. Am F Physiol 1995;268:G128-33.
Mittal RK, Fisher MJ. Electrical and mechanical inhibition of the crural diaphragm during transient relaxation of the of the crural diaphragm during transient relaxation of the
lower esophageal sphincter. Gastroenterology 1990;99: 1265-8.

14 Salapatek AMF, Diamant NE. Assessment of neural inhibition of the lower esophageal sphincter in cats with esophagitis. Gastroenterology 1993;104:810-18.

15 Chakraborty TK, Ogilvie AL, Heading RC, et al. Abnormal cardiovascular reflexes in patients with gastro-oesophageal reflux. Gut 1989;30:46-9.

16 Cunningham KM, Horowitz M, Riddel PS, et al. Relations among autonomic nerve dysfunction, oesophageal motility and gastric emptying in gastro-oesophageal reflux disease. Gut 1991;32:1436-40.

17 Ogilvie AL, James PD, Atkinson M. Impairment of vagal function in reflux oesophagitis. $Q \mathcal{F}$ Med 1985;213:61-74.

18 Sloan S, Rademaker AW, Kahrilas PJ. Determinants of gastroesophageal junction incompetence: hiatal hernia, lower troesophageal junction incompetence: hiatal hernia, lower 977-82.

19 Lind JF, Crispin JS, McIver DK. The effect of atropine on the gastroesophageal sphincter. Can $\mathcal{F}$ Physiol Pharmacol 1968;46:233-8

20 Dodds W, Dent J, Hogan W, et al. Effect of atropine on esophageal motor functions in humans. Am F Physiol 1981; 240:G290-6.

21 Skinner DB, Camp TF. Relation of esophageal reflux to ower esophageal sphincter pressures decreased by atropine. Gastroenterology 1968;54:543-51.

22 Dow TG, Brock-Utne JG, Rubin J, et al. The effect of atropine on the lower esophageal sphincter in late pregnancy. Obstet Gynecol 1978;51:426-30.

23 Parys V, Bruley des Varannes S, Ropert A, et al. Use of an electronic barostat for measurement of motor response of the proximal stomach to feeding and different nervous the proximal stomach to feeding and different ner

24 Bruley des Varannes S, Parys V, Ropert A, et al. Erythromycin enhances fasting and postprandial proximal gastric tone in humans. Gastroenterology 1995;109:32-9.

25 Mittal RK, Chiareli C, Liu J, et al. Atropine inhibits gastric distension and pharyngeal receptor mediated lower oesophageal sphincter relaxation. Gut 1997;41:285-91.

26 Lidums I, Bermingham H, Holloway R. Effect of hyoscine butylbromide on gastroesophageal reflux and lower esophageal sphincter function in healthy volunteers [abstract]. Gastroenterology 1997;112:A778.

27 Mittal RK, Holloway RH, Penagini R, et al. Transient lower esophageal sphincter relaxation. Gastroenterology 1995;109: $601-10$. 